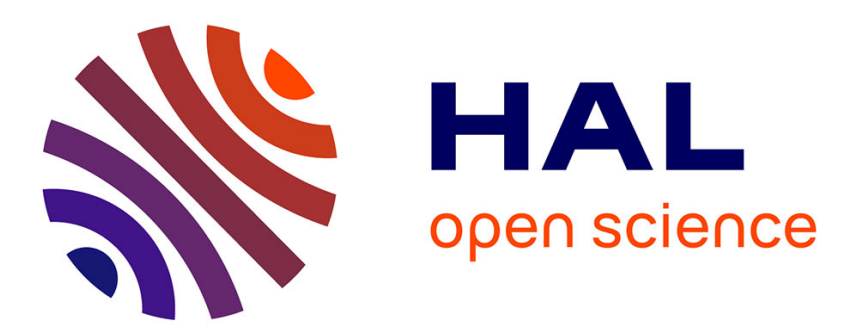

\title{
Mise en évidence d'un champ électrique critique dans le comportement pyroélectrique de T. G. S.
}

\author{
B. Jannot
}

\section{To cite this version:}

B. Jannot. Mise en évidence d'un champ électrique critique dans le comportement pyroélectrique de T. G. S.. Revue de Physique Appliquée, 1974, 9 (6), pp.955-960. 10.1051/rphysap:0197400906095500 . jpa-00243865

\section{HAL Id: jpa-00243865 https://hal.science/jpa-00243865}

Submitted on 1 Jan 1974

HAL is a multi-disciplinary open access archive for the deposit and dissemination of scientific research documents, whether they are published or not. The documents may come from teaching and research institutions in France or abroad, or from public or private research centers.
L'archive ouverte pluridisciplinaire HAL, est destinée au dépôt et à la diffusion de documents scientifiques de niveau recherche, publiés ou non, émanant des établissements d'enseignement et de recherche français ou étrangers, des laboratoires publics ou privés. 


\title{
REVUE DE PHYSIQUE APPLIQUÉE
}

\author{
Supplément au « Journal de Physique *
}

\begin{abstract}
Classification
Physics Abstracts

8.770

\section{MISE EN ÉVIDENCE D'UN CHAMP ÉLECTRIQUE CRITIQUE DANS LE COMPORTEMENT PYROÉLECTRIQUE DE T. G. S.}

\author{
B. JANNOT \\ Laboratoire de Diélectriques, Faculté des Sciences MIPC, \\ Bâtiment Mirande, 21000 Dijon, France
}

(Reçu le 19 novembre 1973, révisé le 2 avril 1974)

\begin{abstract}
Résumé. - Une nouvelle méthode dynamique de mesure du signal pyroélectrique a été appliquée à l'étude de l'action d'un champ électrique sur le comportement pyroélectrique d'un échantillon de TGS, dont la température moyenne est stabilisée. La technique de corrélation et l'emploi d'amplificateurs à très faible bruit donnent à notre détecteur un très bon rapport signal sur bruit. Nous avons par cette méthode montré l'existence d'un champ électrique critique $E_{\mathrm{cr}}$ en dessous duquel les parois de domaines sont bloquées, et nous indiquons la valeur minimum $E_{\mathrm{m}}$ du champ nécessaire pour rendre le cristal monodomaine (10 réf.).
\end{abstract}

Abstract. - In this paper, a new dynamical method for pyroelectric measurement is used to investigate the effect of a biasing field on the pyroelectric behavior of a single crystal of TGS held at different stabilized temperatures. The correlation technique and the use of very low noise amplifiers for our detector result in very good signal to noise ratios. With this method, we show the existence of a critical field $E_{\mathrm{cr}}$ which is necessary to move the domain walls, and a minimal field $E_{\mathrm{m}}$ which is required to produce a single domain state.

1. Introduction. - L'action d'un champ électrique sur le comportement pyroélectrique du sulfate de glycocolle (TGS) a été étudiée par plusieurs auteurs, en particulier Chynoweth [1,2], qui a montré que, dans un ferroélectrique, le coefficient $p=\mathrm{d} P / \mathrm{d} T$ était une fonction décroissante du champ.

Récemment, en utilisant une technique différente de celle de Chynoweth, Micheron [3] a montré que ce coefficient était une fonction croissante pour les champs faibles, et qu'il ne devenait une fonction décroissante que pour les champs forts.

C'est dans le but de préciser ce dernier résultat que notre étude a été entreprise.

2. La méthode expérimentale. - 2.1 PRINCIPE. Toutes les mesures ont été effectuées à l'équilibre thermique. La méthode dynamique mise au point par Chynoweth [1] n'a pu être retenue dans sa forme simple en raison des faibles variations du signal pyroélectrique que nous avons été amené à mesurer, ces petits signaux étant masqués par le bruit (bruit thermique du cristal, bruit des amplificateurs). La technique de corrélation, associée à la méthode dynamique, nous a permis d'extraire le signal du bruit, et ainsi d'augmenter la précision des mesures.

Sur une plaquette de TGS monocristallin, taillée perpendiculairement à l'axe ferroélectrique, on dépose, sur une face, une électrode uniforme et sur l'autre face, deux électrodes semi-circulaires, de façon à constituer deux condensateurs plans (Fig. 1). Deux résistances de charge $R_{1}$ et $R_{2}$ sont respectivement placées aux bornes de ces deux capacités.

$\mathrm{Ce}$ cristal est fixé sur un thermostat maintenu à la température $T$. Afin de créer une petite variation périodique $\mathrm{d} T$, de sa température, autour de la valeur moyenne $T$, on soumet l'échantillon à un rayonnement infra-rouge modulé. Cette variation périodique de la température provoque une variation périodique $\mathrm{d} P$ de la polarisation totale du cristal, autour de la valeur moyenne $P(T)$, ce qui donne naissance, par effet pyroélectrique, à deux tensions $v_{1}(t)$ et $v_{2}(t)$ respectivement aux bornes de $\boldsymbol{R}_{\mathbf{1}}$ et $\boldsymbol{R}_{\mathbf{2}}$.

Le courant pyroélectrique prenant naissance dans une capacité s'écrit :

$$
i=A \cdot \frac{\mathrm{d} P}{\mathrm{~d} t}
$$

où $A$ désigne l'aire d'une demi-électrode frontale. On peut faire intervenir le coefficient pyroélectrique du matériau à la température $T$, et écrire :

$$
i(T)=p(T) \cdot A \cdot \frac{\mathrm{d} T}{\mathrm{~d} t}
$$

avec $p(T)=\mathrm{d} P(T) / \mathrm{d} T$.

La figure 2 représente le schéma électrique équivalent à l'une des capacités, abstraction faite des sources 


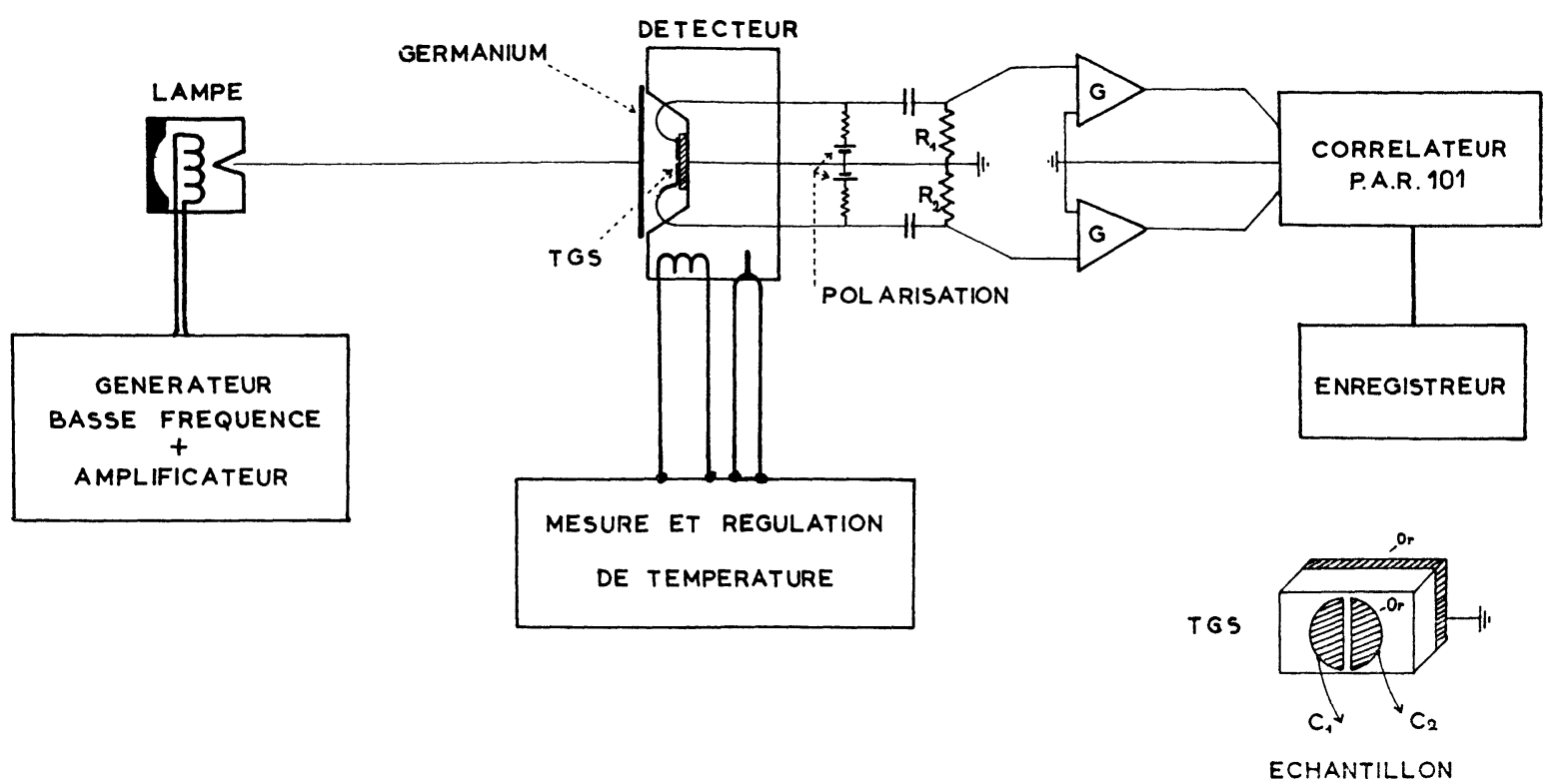

FIG. 1. - Dispositif expérimental ; disposition des électrodes sur l'échantillon.

T.G.S.

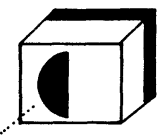

$\mathrm{C}_{1}$
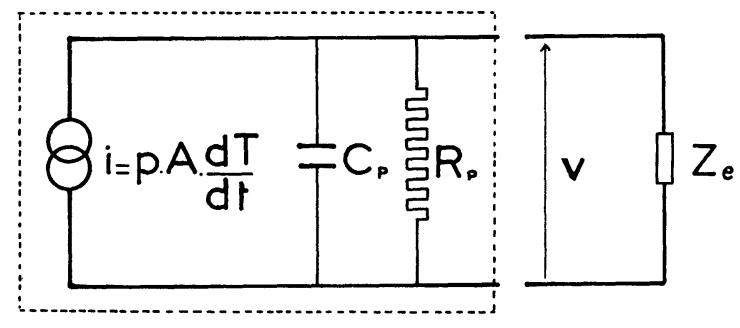

ÉLÉMENT PYROÉLECTRIQUE

FIG. 2. - Schéma électrique équivalent à l'une des capacités (abstraction faite des sources de bruit). $C_{\mathrm{p}}$ est la capacité $; \boldsymbol{R}_{\mathrm{p}}$ la résistance équivalente aux pertes, à la fréquence de mesure. $Z_{\mathrm{e}}$ est l'impédance d'entrée d'un préamplificateur.

de bruit. La tension $v$ apparaissant à l'entrée de l'amplificateur de résistance d'entrée $R_{\mathrm{e}}$ est solution de l'équation :

$$
i=C_{\mathrm{p}} \cdot \frac{\mathrm{d} v}{\mathrm{~d} t}+\frac{v}{R_{\mathrm{T}}}=p(T) \cdot A \cdot \frac{\mathrm{d} T}{\mathrm{~d} t}
$$

où l'on a posé :

$$
R_{\mathrm{T}}^{-1}=R_{\mathrm{p}}^{-1}+R_{\mathrm{e}}^{-1}
$$

$R_{\mathrm{p}}$ et $C_{\mathrm{p}}$ étant les éléments de l'impédance du schéma parallèle équivalent à une capacité.

Le flux infra-rouge reçu par le cristal étant modulé sinusoïdalement, à la pulsation $\omega$, la variation de température $\mathrm{d} T$ qu'il engendre est elle-même sinusoïdale, et l'équation (3) admet une solution sinusoï- dale, qui s'écrit, dans le cas pratique où $t$ est très supérieur à $R_{\mathrm{T}} . C_{\mathrm{p}}$ et où les résistances de charge des deux capacités sont très inférieures aux résistances $R_{\mathrm{p} 1}$ et $R_{\mathrm{p} 2}$ (cf. Fig. 2) :

$$
v=K \cdot p(T) \cdot \cos (\omega t-\varphi) .
$$

$K$ est indépendant de la température et du champ de polarisation $E$, si l'on néglige les anomalies de chaleur spécifique et de diffusivité thermique à la transition.

Les deux capacités étant identiques, les deux tensions apparaissant aux bornes de $R_{\mathrm{e} 1}$ et $R_{\mathrm{e} 2}$ sont identiques :

$$
v_{1}=v_{2} \text {. }
$$

En fait, ces deux tensions ne sont pas directement accessibles à la mesure, car on doit respectivement leur ajouter les tensions de bruit thermique $b_{1}(T)$ et $b_{2}(T)$ de chacune des capacités en parallèle sur sa résistance de charge.

La corrélation temporelle permet d'extraire le signal du bruit. Les deux tensions $v_{1}$ et $v_{2}$ sont séparément amplifiées et filtrées (la bande passante est très étroite puisque les tensions sont sinusoïdales). Désignons par $V_{1}$ et $V_{2}$ les tensions ainsi obtenues.

Les deux électrodes frontales placées sur l'échantillon sont suffisamment proches pour recevoir uniformément le flux infra-rouge, mais leur distance est encore très supérieure à la longueur de corrélation des fluctuations, de sorte que les bruits thermiques prenant naissance dans chaque capacité sont indépendants donc non corrélés.

D'autre part, les résistances de charge $R_{1}$ et $R_{2}$, les sources de polarisation de chaque capacité, les deux amplificateurs et filtres sont indépendants. Si $a_{1}$ et $a_{2}$ désignent les bruits respectifs des deux 
chaînes de mesure de gain total $G$, la bande passante étant très étroite, on peut écrire :

$$
\begin{aligned}
& V_{1}=\left(v_{1}+b_{1}\right) \cdot G+a_{1} \\
& V_{2}=\left(v_{2}+b_{2}\right) \cdot G+a_{2} .
\end{aligned}
$$

Ces deux tensions sont envoyées sur les deux entrées d'un corrélateur qui effectue, en temps réel, la moyenne temporelle de leur produit, et donne la fonction d'intercorrélation de $V_{1}$ et $V_{2}$, définie par:

$$
C_{V_{1} V_{2}}(\tau)=\lim _{t \rightarrow \infty} \frac{1}{t} \int_{0}^{t} V_{1}(t) \cdot V_{2}(t-\tau) \mathrm{d} t .
$$

En remplaçant dans (7), $V_{1}$ et $V_{2}$ par leurs expressions données par (6), et en vertu de la linéarité de l'opération de corrélation, $C_{V_{1} V_{2}}(\tau)$ se décompose en une somme de neuf fonctions de corrélation qui sont toutes nulles, d'après ce qui précède, sauf celle où apparaît le produit des deux tensions d'origine purement pyroélectrique $v_{1}(t)$ et $v_{2}(t)$, puisque l'on suppose que la variation de température sinusoïdale $\mathrm{d} T$ qui leur donne naissance est la même en tous les points du cristal. On vérifie d'ailleurs expérimentalement qu'en absence de flux infra-rouge, $C_{V_{1} V_{2}}(\tau)$ est nulle, ce qui justifie l'hypothèse précédente de la non-corrélation des fluctuations.

En remplaçant $v_{1}$ et $v_{2}$ par leurs expressions (4), la fonction d'intercorrélation de $V_{1}$ et $V_{2}$ s'écrit :

$$
C_{V_{1} V_{2}}(\tau)=\frac{1}{2} \cdot G^{2} \cdot p^{2} \cdot K^{\prime 2} \cdot \cos \omega \tau
$$

soit :

$$
C_{V_{1} V_{2}}(\tau)=S(T, E) \cdot \cos \omega \tau .
$$

Avec les hypothèses faites précédemment, $K^{\prime}$ ne dépend ni de $T$ ni de $E$. Il en résulte que la dépendance de $S(T, E)$ avec $T$ et $E$ est due principalement au terme en $p^{2}$. Les variations de $p$ avec $T$ et $E$ sont donc reproduites, à une constante près, par celles de $\sqrt{S}$.

2.2 LE DISPOSITIF EXPÉRIMENTAL. - L'échantillon de T.G.S., coupé dans le monocristal, est poli jusqu'à une épaisseur de $300 \mu$. Les électrodes sont déposées sur le cristal par évaporation d'or, elles sont semi-circulaires, ont $4 \mathrm{~mm}$ de diamètre, et leurs centres sont distants de $0,2 \mathrm{~mm}$. L'épaisseur de la couche d'or a été fixée à $150 \AA$ [4] afin que l'électrode soit transparente au rayonnement infrarouge, tout en étant conductrice et peu réfléchissante. Deux fils d'argent très fins, fixés sur chacune des deux demi-électrodes par un point de laque d'argent, relient ces électrodes aux entrées des amplificateurs (Fig. 1).

Le thermostat sur lequel est collé l'échantillon est constitué par un disque de cuivre de $5 \mathrm{~mm}$ d'épaisseur et de $1,5 \mathrm{~cm}$ de diamètre, l'ensemble étant placé à l'intérieur d'un four en cuivre stabilisé par une régulation électronique. $\mathrm{La}$ température d'équilibre du cristal est mesurée par un thermomètre électronique. Une fenêtre de germanium placée dans la paroi frontale du four sert de filtre pour la radiation infrarouge et assure la continuité du blindage électrique. Le rayonnement infra-rouge, modulé à $30 \mathrm{~Hz}$, est produit par une lampe alimentée par un courant de basse fréquence $(15 \mathrm{~Hz})$ et est focalisé sur le cristal par un miroir concave.

Chaque amplificateur sélectif, de gain total $G$, est constitué tout d'abord d'un préamplificateur, alimenté par des piles, et placé immédiatement après le cristal, puis d'un amplificateur P. A. R. modèle 101, dont la bande passante est très étroite, et centrée sur $30 \mathrm{~Hz}$.

L'étage d'entrée des préamplificateurs est constitué par un transistor à effet de champ qui a été trié pour son faible bruit, et dont la résistance de polarisation de grille sert de résistance de charge. La capacité d'entrée, de l'ordre de $2,5 \mathrm{pF}$, peut être négligée.

Enfin, deux sources de tension indépendantes polarisent respectivement chaque capacité.

Le four contenant le cristal, les sources de polarisation et les préamplificateurs sont placés dans une enceinte en duralumin assurant un bon blindage électrique. Four, préamplificateurs et régulation de température ont été construits au Laboratoire.

3. Résultats expérimentaux. - Nous avons étudié les variations du coefficient pyroélectrique $p$, en fonction de la température et du champ électrique appliqué, au voisinage de la température de transition $\left(T_{0}=49,5^{\circ} \mathrm{C}\right)$. Avant chaque série de mesures (soit une courbe $p=f(E)$ pour $T$ fixé), on commence par rendre l'échantillon polydomaine (rajeunissement). Pour cela, l'échantillon polarisé est porté à $70^{\circ} \mathrm{C}$ pendant une heure environ, puis, après l'avoir mis en court-circuit, on le ramène en phase ferroélectrique à la température de mesure.

L'échantillon est ensuite soumis à des champs continus $E$ croissants. La figure 3 donne les résultats de mesure.

Pour une température donnée, on observe les résultats suivants (Fig. 4) :

Lorsque le champ appliqué $E$ est inférieur à une valeur critique $E_{\mathrm{cr}}$, le coefficient $p$ reste pratiquement constant, sauf au voisinage immédiat de $T_{0}$ où l'on observe une légère diminution. Lorsque $E$ est compris entre $E_{\mathrm{cr}}$ et une valeur $E_{\mathrm{m}}$, on observe un fort accroissement de $p$. Pour $E$ supérieur à $E_{\mathrm{m}}, p$ décroît quand le champ augmente ; cette décroissance est plus forte lorsque $T$ est voisin de $T_{0}$.

Le minimum de $p$ observé pour les champs appliqués inférieurs à $E_{\mathrm{cr}}$ n'est pas reproductible et dépend de la façon dont le cristal réagit à la trempe. Par contre le maximum de $p$ pour $E=E_{\mathrm{m}}$ est reproductible, ainsi que les valeurs de $E_{\mathrm{cr}}$ et $E_{\mathrm{m}}$.

Lorsqu'on fait décroître le champ appliqué à partir d'une valeur supérieure à $E_{\mathrm{m}}$, le coefficient $p$ croît jusqu'à la valeur maximale qu'il conserve jusqu'à $E=0$. 


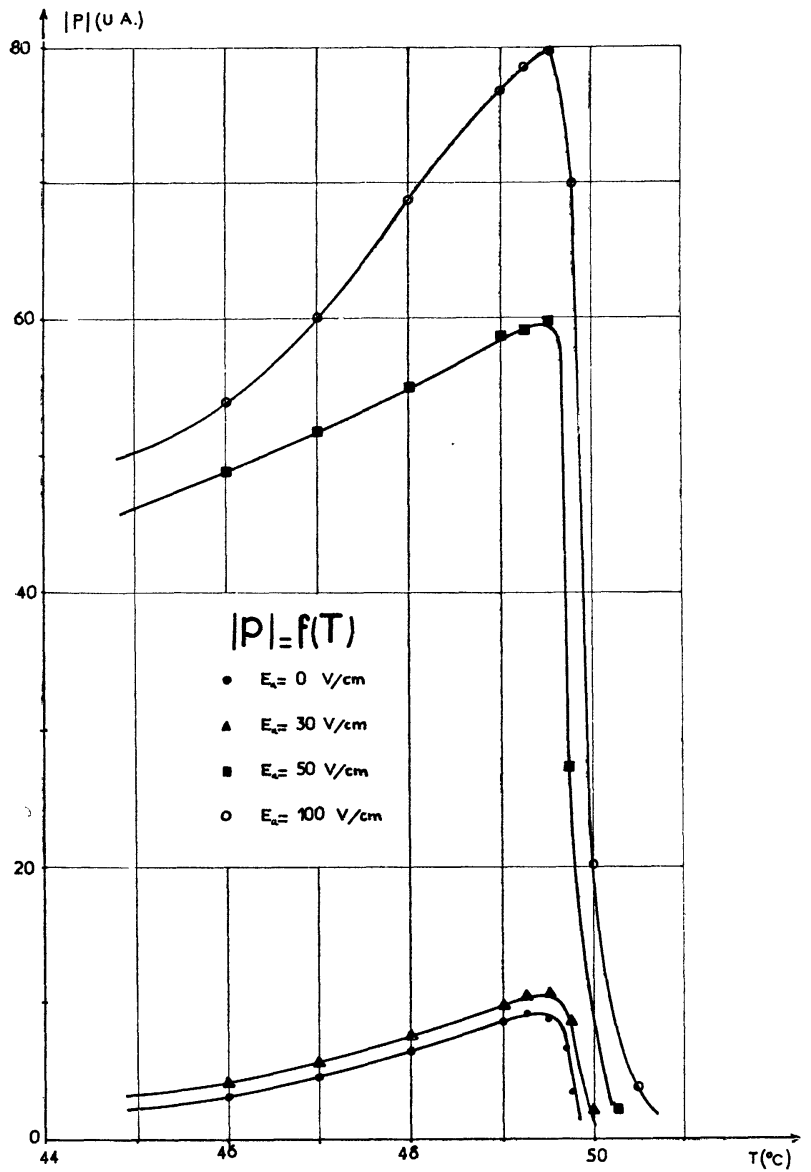

FIG. 3. - Variations du coefficient pyroẻlectrique de TGS avec la température, pour différentes valeurs du champ de polarisation.

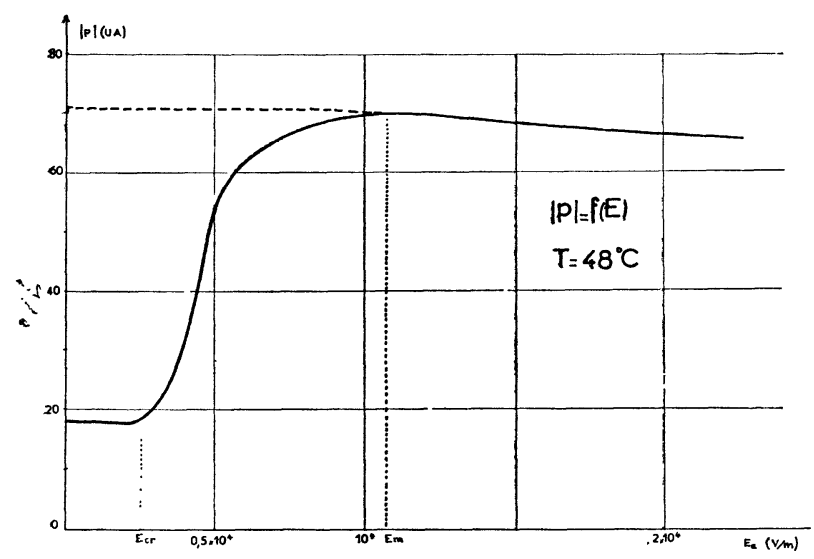

FIG. 4. - Variations du coefficient pyroélectrique de TGS avec le champ appliqué, pour une température donnée $\left(T=48^{\circ} \mathrm{C}\right)$.

Les figures 5 et 6 donnent les résultats expérimentaux à la température de transition $T_{0}$ (la figure 6 est un agrandissement de la figure 5 pour les champs faibles).

Si l'on change le sens de la polarisation et que l'on effectue les mêmes opérations (y compris le traitement thermique préalable), les courbes $p=f(E)$ obtenues sont symétriques des précédentes par rapport à l'axe $E=0$.

Nous mettons donc ainsi en évidence trois domaines de champ où le comportement pyroélectrique de TGS est différent.

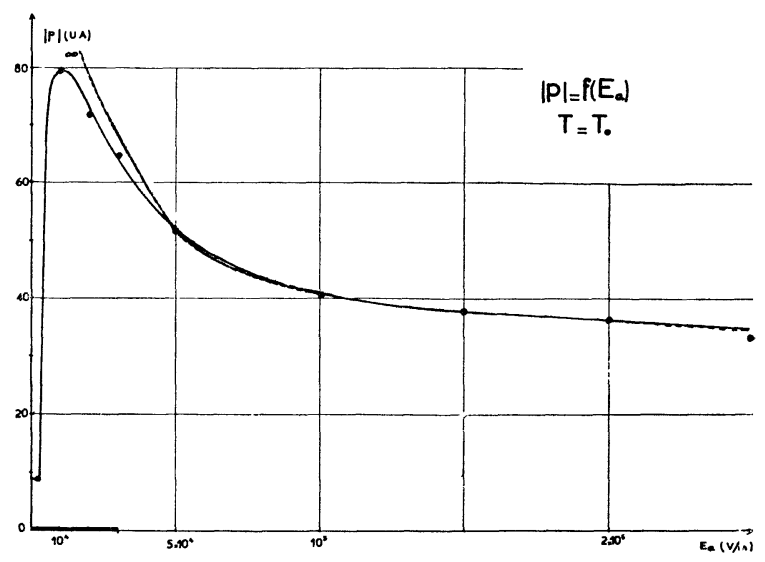

Fig. 5. - Variation du coefficient pyroélectrique de TGS avec le champ appliqué, à la température de Curie $T=T_{0}$. Le domaine de champ indiqué en trait plus soutenu est détaillé sut la figure 6.

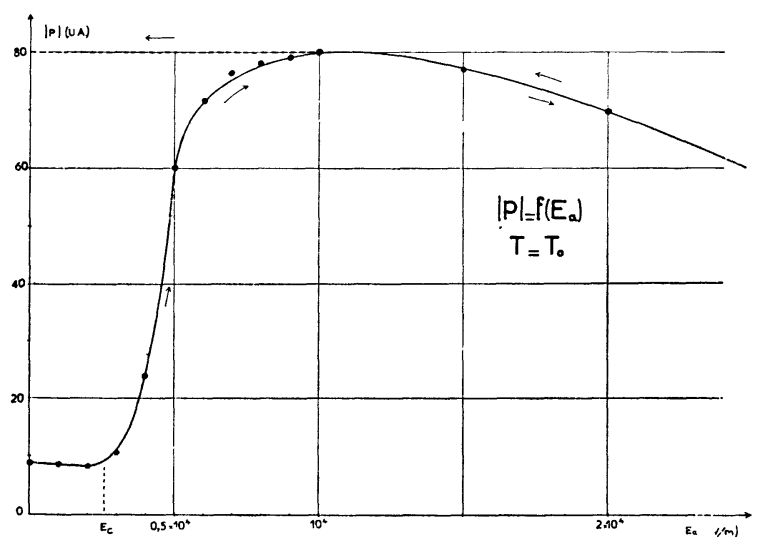

Fig. 6. - Agrandissement de la figure 5 pour les champs faibles. Les flèches indiquent le sens de variation du champ.

Suivant les échantillons étudiés, les valeurs de $E_{\mathrm{cr}}$ varient entre 20 et $50 \mathrm{~V} / \mathrm{cm}$, celles de $E_{\mathrm{m}}$ varient entre 100 et $200 \mathrm{~V} / \mathrm{cm}$.

4. Interprétation et discussion des résultats. 4.1 ChAMPS Supérieurs a $E_{\mathrm{m}}$. - Dans ce cas, le cristal est monodomaine et on peut interpréter les résultats observés par la théorie thermodynamique [6].

En prenant pour l'énergie libre le développement simplifié :

$$
F=F_{0}+\frac{1}{2} \cdot \beta \cdot\left(T-T_{0}\right) \cdot P^{2}+\frac{1}{4} \cdot \gamma \cdot P^{4}
$$


on déduit :

$$
E=\left(\frac{\partial F}{\partial P}\right)_{T}=\beta \cdot\left(T-T_{0}\right) \cdot P+\gamma \cdot P^{3} .
$$

De cette dernière équation, nous avons déduit les courbes $P=f(T)$ pour différentes valeurs 'du champ appliqué (en prenant pour $\beta$ et $\gamma$ les valeurs données par Triebwasser [7]:

$$
\left.\beta=3,92 \times 10^{-3} \mathrm{cgs}, \quad \gamma=8 \times 10^{-10} \mathrm{cgs}\right) .
$$

En différentiant (10), on obtient:

$$
\mathrm{d} E=0=\left[\beta \cdot\left(T-T_{0}\right)+3 \cdot \gamma \cdot P^{2}\right] \cdot \mathrm{d} P+\beta \cdot P \cdot \mathrm{d} T
$$

d'où l'on déduit :

$$
p=\frac{\mathrm{d} P}{\mathrm{~d} T}=\frac{-\beta \cdot P}{\beta \cdot\left(T-T_{0}\right)+3 \cdot \gamma \cdot P^{2}}
$$

où $P$, polarisation à la température $T$ lorsqu'un champ $E$ est appliqué, est donné par le réseau de courbes $P=f(T)$.

En particulier, à la température de transition, on déduit de (10) :

$$
P=\left(\frac{E}{\gamma}\right)^{1 / 3}
$$

ce qui donne :

$$
|p|=\frac{\beta}{3 \cdot \gamma \cdot \cdot^{2 / 3}} \cdot E^{-1 / 3} .
$$

Sur la figure 7 , nous comparons les résultats expérimentaux avec la courbe théorique en ajustant le point correspondant à $E=2 \mathrm{kV} / \mathrm{cm}$, pour $T=T_{0}$.

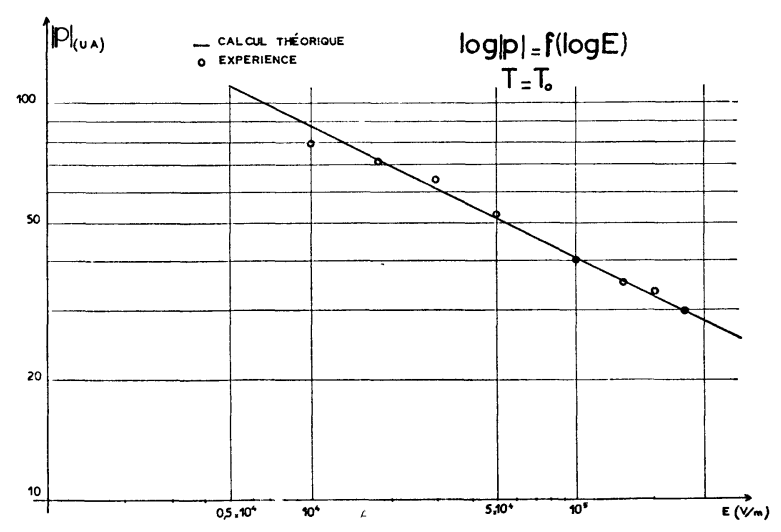

Fig. 7. - Variations du coefficient pyroélectrique avec le champ appliqué pour $T=T_{0}$. Le trait uniforme indique les variations théoriques calculées en ajustant le point correspondant à $E=2 \mathrm{kV} / \mathrm{cm}$. Les cercles sont les points expérimentaux.

Nous constatons que l'accord est très satisfaisant pour les champs forts, c'est-à-dire lorsque le cristal est monodomaine.

4.2 ChAmps inférieurs a $E_{\mathrm{m}}$ - En ce qui concerne le comportement de TGS pour les champs faibles, nous avons rapproché nos résultats de ceux obtenus par Stankowska et Krysinska [8] qui ont étudié l'influence d'un champ électrique sur la structure en domaines de TGS. Ils ont montré, en observant directement les domaines, aux températures $40^{\circ}, 45^{\circ}$ et $48^{\circ} \mathrm{C}$, que le champ minimum nécessaire pour rendre monodomaine un cristal de TGS rajeuni est de l'ordre de $100 \mathrm{~V} / \mathrm{cm}$ pour un cristal pur et varie de 100 à $2400 \mathrm{~V} / \mathrm{cm}$ pour un cristal contenant des impuretés. Ils signalent d'autre part que dans les cristaux purs, le mouvement des parois de domaines ne se produit que lorsque le champ appliqué dépasse une valeur de l'ordre de $20 \mathrm{~V} / \mathrm{cm}$. Un tel champ minimum a également été mis en évidence dans TGS par Giletta [10], et dans KDP par Bornarel et Lajzerowicz [9].

Il est très vraisemblable que le champ critique que nous avons mis en évidence par effet pyroélectrique, résulte de la structure en domaines de TGS dans la phase ferroélectrique. En effet, si on désigne par $f$ la fraction de domaines pour lesquels la polarisation a un certain sens, la polarisation totale de l'échantillon s'écrit :

$$
P=f \cdot P_{\mathrm{s}}-(1-f) \cdot P_{\mathrm{s}}=(2 f-1) \cdot P_{\mathrm{s}}
$$

d'où la valeur du coefficient pyroélectrique :

$$
p=\frac{\partial P}{\partial T}=(2 f-1) \cdot \frac{\mathrm{d} P_{\mathrm{s}}}{\mathrm{d} T}
$$

(la température varie suffisamment peu au cours de la mesure pour que $f$ n'en dépende pas).

Pour un cristal rajeuni, $f$ est voisin de $\frac{1}{2}$ et par conséquent $p$ est faible. Sous l'effet d'un champ électrique, $f$ tend vers 1 et le coefficient pyroélectrique prend la valeur maximale correspondant au cas d'un cristal monodomaine.

Nos mesures n'ont pas expliqué l'origine du champ critique dans TGS. Toutefois, des travaux antérieurs nous permettent de supposer qu'il est dû à l'existence de défauts (défauts polarisés, ou dislocations), ayant pour effet de bloquer les parois de domaines; celles-ci ne peuvent alors être mises en mouvement que sous l'action d'un champ d'amplitude au moins égale à $E_{\mathrm{cr}}$, cette valeur étant d'autant plus faible que le cristal est plus pur.

5. Conclusion. - Par une méthode d'analyse précise, nous avons étudié l'action d'un champ électrique sur TGS, et principalement l'action des champs faibles. Outre l'existence d'un champ critique $E_{\mathrm{cr}}$, nous avons montré que l'application d'un champ $E_{\mathrm{m}}$, très faible, suffisait pour rendre et conserver un échantillon monodomaine, et ainsi garder au coefficient pyroélectrique, sa valeur maximale.

Remerciements. - Que M. le Professeur Godefroy et $\mathrm{M}$. Micheron trouvent ici l'expression de nos remerciements pour l'aide qu'ils ont apportée à la réalisation de ce travail. 


\section{Bibliographie}

[1] Chynoweth, A. G., J. Appl. Phys. 27 (1958) 78.

[2] Chynoweth, A. G., Phys. Rev. 117 (1960) 1235.

[3] Micheron, M., Godefroy, L., C. R. Hebd. Séan. Acad. Sci. B 273 (1971) 143.

[4] Stanford Jr, A. L., Solid State Electron. 8 (1965) 747.

[5] Smith, R. A., Jones, F. E., Chasmar, R. P., The detection and measurement of infra-red radiation (Clarendon Press, Oxford) 1968, p. 50.
[6] Devonshire, A. F., Phil. Mag. 40 (1949) 1040.

[7] Triebwasser, S., IBM. J. Res. \& Develop. (1958) 212.

[8] Stankowska, J., Krysinska, M., Acta Phys. Pol. A 40 (1971) 239.

[9] Bornarel, J., LaJzerowicz, J., J. Physique Collq. 33 (1972) C2-151.

[10] Gilletta, F., C. R. Hebd. Séan. Acad. Sci., 264B (1967) 400. 